\title{
A cross-sectional study examining predictors of visit frequency to local green space and the impact this has on physical activity levels
}

Elliott P. Flowers', Paul Freeman and Valerie F. Gladwell

\begin{abstract}
Background: Lack of physical activity (PA) is a growing public health concern. There is a growing body of literature that suggests a positive relationship may exist between the amount of local green space near one's home and PA levels. For instance, park proximity has been shown to predict PA levels amongst certain populations. However, there is little evidence for the role of relatedness towards nature and perceptions of local green space on this relationship. The aim of this study was to examine, in a National UK sample, whether subjective indices associated with local green space were better predictors of visit frequency to local green space and PA levels compared to objectively measured quantity of local green space.
\end{abstract}

Methods: A cross-sectional survey was designed. From a random sample, 2079 working age adults responded to an online survey in September 2011. Demographics, self-reported PA, objective measures of the local environment (including local green space, road coverage, and environmental deprivation), were assessed in conjunction with perceptions of local green space and nature relatedness. Quantity of local green space was assessed by cross-referencing respondents' home postcodes with general land use databases. Regression models were conducted to assess which of our independent variables best predicted visit frequency to local green space and/or meeting PA guidelines. In addition, an ordinal regression was run to examine the relationship between visit frequency to local green space and the likelihood of meeting national PA guidelines.

Results: Nature relatedness was the strongest predictor for both visit frequency to local green space and meeting PA guidelines. Results show that perceived quality is a better predictor of visit frequency to local green space than objective quantity of local green space. The odds of achieving the recommended amount of PA was over four times greater for people who visited local green space once per week compared to never going (OR 4.151; $95 \% \mathrm{Cl}$, 2.40 to 7.17$)$.

Conclusions: These results suggest that perceptions of local green space and nature relatedness play an important role in the relationship between local green space and PA. Considering the known health benefits of PA, our results are potentially important for public health interventions, policy making and environmental planning.

Keywords: Local green space, Nature relatedness, Perceptions, Physical activity, Green exercise

\footnotetext{
*Correspondence: eflowe@essex.ac.uk

School of Biological Sciences, University of Essex, Colchester CO4 3SQ, UK
} 


\section{Background}

Physical activity (PA) is a well-known contributor to good health [1]. Current guidelines for aerobic activity recommend that adults should spend at least 150 min per week in moderately intensive PA or 75 min of vigorous PA (or any combination of the two) [2, 3]. In the most recent Health Survey for England, unfortunately, only $67 \%$ of men and $55 \%$ woman met the recommended guidelines [4]. This is replicated to a lesser extent throughout the whole world, with $20 \%$ of men and $27 \%$ of women considered not to meeting the guidelines [5]. Therefore, increasing levels of PA is a major priority in Public Health, particularly in Westernised countries [6].

An increasing number of studies have investigated the impact of the natural environment on PA behaviours and health. Systematic reviews have found that there is a positive correlation between the availability of local green space (LGS) and PA levels [7-9]. The mechanisms for this, however, remain unclear.

Recently, a schematic model of the motivational processes underlying the relationship between natural environments and PA was proposed [10]. In a compelling argument, the authors suggested two distinct motivational pathways for visiting LGS: firstly the active use of natural environment and secondly as a contributor to active living. Consistent with these pathways, this study explores how feelings about nature influence visits to LGS (active use) and the subsequent relationship this has on PA (active living). Specifically, we examine the influence of both objective and subjective measures of the local environment on visit frequency to LGS and the likelihood of meeting PA guidelines. We also explore the influence of visit frequency to LGS on PA levels.

The literature in this area has predominately focused on two examples of green spaces, namely natural environments and urban green spaces, or a combination of the two [11]. By definition, natural environments are those that occur naturally on earth. They differ from urban green spaces in that they have had minimum human input in their design, creation, and maintenance [11]. Both are used as locations for recreational activities in modern society. For the purposes of this study, LGS is a combination of urban green space and natural environments in close proximity to the home.

Many recreational activities that take place in LGS involve some form of PA such as walking, jogging, and play [12-14]. Even less intense activities like photography, reading, and fishing often require individuals to walk to desired locations. Thus, visit frequency to LGS may be positively associated with overall PA levels and subsequently the likelihood of meeting PA guidelines.

A recent review of the impact of LGS on PA found that there is a huge variety of research methods employed within the field, including objective and subjective measures of LGS [10]. Studies using objective measures have predominately focused on specific locations and used a Geographical Information System (GIS) to assess LGS [15-17]. Using GIS, researchers can analyse geographical data and categorise into various land uses (domestic buildings, roads, green space etc.). Conversely, subjective measures embrace self-report questionnaires to provide vital insight into individuals' perceptions of LGS (pLGS). Requiring fewer resources, subjective measures enable investigation of some variables over much larger geographic areas [18, 19]. Interestingly, when both objective and subjective measures have been used to determine quantity of LGS in the same geographical area contemporaneously, discrepancies have been found between perceptions of park proximity and actual distance to park [20] as well as perceived versus actual quantity [21].

To date, only a small number of studies have investigated the relationships between objectively measured LGS, PA, and health on a national scale [22-25]. These studies have found mixed results. For example, in the Netherlands, the quantity of LGS within a one $\mathrm{km}$ radius of home address was associated with 15 indicators of wellbeing [22]. In contrast, neighbourhood park access was not associated with body mass index (BMI) in New Zealand, although beach access was related to BMI [25].

Furthermore, two studies in the UK have also produced mixed results. In England, individuals who lived in the greenest quintile of England were 1.27 times (95 \% CI, 1.13 to 1.44 ) more likely to meet PA guidelines than individuals in the least green quintile [23]. In contrast, no association was found between LGS and meeting PA guidelines in Scotland [25].

A systematic review [10] suggested that perceptions or subjective measures of LGS access are stronger predictors of PA than environmental barriers such as actual proximity (e.g., $[18,26])$. For example, perceived access to LGS has been linked with PA levels in Canada [20] and Australia [27]. In the UK, a few localised studies have investigated the relationship between pLGS and green space usage (e.g., [19, 28]). Results from Oxford and Bristol found that the majority of people were satisfied with accessibility to LGS. In Bristol, however, despite good perceived access, only $31 \%$ of participants visited LGS on a weekly basis. This suggests that other factors are likely to play a crucial role in the actual use of LGS and in turn PA levels.

Beyond the role of perceived access, it is important to consider the perceived quality of LGS. Commonly reported as 'satisfaction with neighbourhood parks', evidence suggests perceived quality of LGS is positively related to PA $[18,29]$. This further highlights the potential importance of pLGS for PA.

In addition to perceptions of quality and access, individuals' self-reported relationship with nature may be a 
crucial determinant of whether they engage in PA in LGS (termed Green Exercise). Evidence from recent studies suggests that nature relatedness (individual levels of connectedness with the natural world; NR) plays an important role in engagement with nature and subsequent benefits $[30,31]$. Indeed, NR has been shown to predict travel distance to parks [30], time spent in gardens [30], and psychological well-being [32]. In their schematic model, Calogiuri et al. [10] proposed that feelings about nature influence intentions to visiting LGS.

In summary, there are a number of factors that influence the relationship between LGS and PA, including actual visits to the LGS. Objective (GIS measured quantity of LGS) and subjective (perceived access and quality) have been shown to predict visit frequency to LGS and overall PA. It is vital, however, to take a more nuanced approach to understand the role of perceptions in the relationship between LGS and PA. No study has investigated which pLGS have greatest impact on visit frequency to LGS and subsequent PA. The aims of the study, therefore, were to examine: 1) which objective and perceptual indices of LGS predict visit frequency to LGS? 2) which objective and perceptual indices of LGS predict whether participants meet PA guidelines? and 3) if visit frequency to LGS predicts whether participants meet PA guidelines? It was hypothesised that perceived access and quality of LGS and NR would be stronger predictors of visit frequency to LGS and PA than objectively measured LGS. It was also hypothesised that the likelihood of meeting PA guidelines will increase in a dose-response pattern with visit frequency to LGS.

\section{Method}

The data used in the present study were extrapolated from a larger research project examining the effects of the environment and exercise on psychological health. Part of the project was conducted using an online questionnaire administered to participants in the 150,000 person Harris Poll panel of Great Britain. The research was approved by the University of Essex Research Ethics Committee and participants provided informed consent. Participants were selected at random from the base sample and invited by email to take part in the survey $(n=22,950)$. Data from the responding sample were collected over a 2 week period in late September 2011. Data collection was closed after 2 weeks as it reached the requested number of respondents.

This process yielded a sample of 2079 working age adults. In the current study, data were available for 1988 working age adults (997 males) ranging from 22 to 65 years $(M=43.19, S D=11.46)$, which is the higher than the UK median of 39 years [33]. Only employed individuals were selected for this research in order to control for the impact of active commuting on visiting
LGS and PA levels; $69.8 \%$ were in full-time employment, $18.1 \%$ were in part-time employment, and $12.2 \%$ were self-employed.

The UK Meteorological Office [34] reported that in July and August 2011, mean temperatures were 0.5 to $1.0{ }^{\circ} \mathrm{C}$ below average across most of the UK. In contrast, during September, 2011 - during data collection - the mean temperatures were around $1.1{ }^{\circ} \mathrm{C}$ above average, making it the sixth warmest September in 100 years. Throughout September, most of England experienced below average rainfall; some parts of Northern England and Scotland, however, received over $50 \%$ more rainfall than average [34].

Self-reported health was assessed with a single item which asked "How would you rate your health in the last month?" Participants responded on a Likert scale from " 1 $=$ Terrible" to "7 Excellent". This was included as a covariate in all statistical analyses alongside age and gender.

Objective representation of the local environment was given as \% of LGS available near home. This was calculated to ward level (primary unit of electoral geography), using participants' home postcodes and Geoconvert (an online geography matching and conversion tool) [35]. For \% of LGS, ward coded data were then entered into a database, available from CRESH.org.uk, which has previously been described [36]. In brief, the database used general land use across England, supplemented with a second database covering Scotland, Northern Ireland, and Wales and the coordination of information placed on the environment database [37]. The database provided specific \% of LGS, including all vegetated areas larger than $5 \mathrm{~m}^{2}$ in area (excluding domestic gardens) for each ward in the UK. Green spaces included ranged from transport verges (narrow strip of land between carriageway and road boundary) and neighbourhood greens, to parks, playing fields and woodlands.

Perceived access to LGS was assessed by asking participants "How easy is it to get to the green space local to your home?" Participants responded from 1 = "Very difficult" to $7=$ "Very easy". Perceived quality of LGS was assessed with a single item that asked "How would you rate the quality of your local accessible green spaces that are close to your home?" Participants responded from 1 $=$ "Terrible" to 7 = "Excellent".

NR was assessed using two sections of the NR Scale (NRS; [38]). The self and experience factors were extrapolated to form the NRS-14. The self and experience factor were used to reflect both how strongly people identify with the natural environment and the attraction people have to nature. The perspective factor of the NRS was excluded as we were not interested in global issues such as conservation and species survival rates. Participants were asked to report how they felt about 14 phrases that described their relationship with nature. 
Examples items included, "Even in the middle of the city, I notice nature around me", and "I am not separate from nature, but part of nature". Participants responded using a Likert scale format ranging from 1 = "disagree strongly" to 5 = "agree strongly". Where appropriate, responses were reversed so that higher scores indicated a greater NR. NR was recorded as a mean of 14 items.

Visit frequency to LGS was assessed by asking participants "How often do you visit the green space closest to your home?" This was rated from $1=$ "Every day" to 7 = "Never visit my LGS or any other green spaces". This score was then reversed scored so that a higher frequency of visits was represented by a higher numerical value. Participants also indicated via multiple choice selection how they usually travelled to LGS, and how long it usually took them.

Self-reported PA levels were recorded using a shortform version of the International Physical Activity Questionnaire (IPAQ-SF, [39]). Participants were required to indicate how many days they undertook PA activity for more than $10 \mathrm{~min}$. Subdomains were vigorous, moderate and walking. Furthermore, participants reported how many hours and minutes they usually spent on these activities on one of those days. Additionally, participants reported how many hours and minutes they would usually spend sitting on a week day.

Raw data were converted into weekly PA levels using IPAQ-SF scoring guidelines [40]. The raw data were calculated into a weekly score described as multiples of the resting metabolic rate (METs). As recommended by IPAQ scoring guidelines, some of the raw data was truncated to reduce potential outliers. Above $180 \mathrm{~min}$ in all categories is considered to be unlikely, suggesting participants' misinterpreted the question. In accordance with guidelines [40], therefore, all moderate minutes that were between 180 and 299 were reduced to 180; those above 299 were divided by seven. Also, vigorous minutes over 180 were divided by seven and walking minutes over 180 were reduced to 180 . For the data analysis, participants were dichotomised according to whether they achieved at least 600 MET.min per week or not. Those participants who achieved below 600 MET.min per week in total were classified as not meeting the current minimum requirements for a healthy lifestyle (in accordance with [41]) and in the low category using IPAQ scoring guidelines [40].

A number of variables were included in the study as covariates: age, subjective health, gender, road coverage, environmental deprivation, and active travel to both work and LGS. Environmental Deprivation (at ward level) was obtained from a database that is available on CRESH.org [42]. In summary, ward level measurements were calculated for a variety of environmental dimensions that impact upon health (air pollution, climate, UV radiation, industrial facilities, and green space). Each ward was given a score from -2 to +3 , with +3 indicating most deprived environments. For this study, scores of environmental deprivation were reversed so that the most deprived areas had the lowest score.

Road Coverage was calculated by cross referencing ward codes against general land use database [43] across England ${ }^{1}$ to give the amount of road coverage in each ward. This was converted to a percentage of the total land area in each ward. For both environmental deprivation and road coverage, participants' home post codes were converted to wards using Geoconvert.

Active travel to work was assessed by asking participants "How do you usually travel to work? Tick all that apply". Any participant who ticked walk or cycle were classified as active commuters. Active travel to LGS was assessed by asking participants "How do you usually travel to your local green space? Tick all that apply" Any participants who ticked walk or cycle were classified as active travellers to LGS.

All data analysis was carried out using IBM SPSS Statistics 20. Three regression models were run. First, an ordinal regression model was run to determine whether objective (\% LGS) and subjective (perceived access, perceived quality, and NR) measures predicted frequency of visits to LGS. Additional demographic, objective, and subjective variables were included as covariates in the model (see Table 1).

Second, a binary logistic regression was run to determine whether objective (\% LGS) and subjective (perceived access, perceived quality, and NR) measures predicted the likelihood of meeting current UK PA guidelines. Additional demographic, objective, and subjective variables were included as covariates in the model (see Table 2).

Finally, another binary logistic regression was run to determine if visit frequency to LGS predicted the likelihood of meeting current UK PA guidelines. Age, gender and health were included as covariates in the third model. Nagelkerke $\mathrm{R}^{2}$ tests were run to assess how much of the variance in the outcomes could be accounted for by the models. Statistical significance was accepted at $p<0.05$ throughout the analyses.

\section{Results}

The 1379 urban wards represented in the study had a mean green space coverage of $52.7 \%$ (95 \% CI, 51.5 to 53.9). This is nearly $10 \%$ lower than the UK national average of $62.6 \%$. Furthermore, the wards had a mean road coverage of $10.1 \%$ (95 \% CI, 3.7 to $16.6 \%$ ) and a mean environmental deprivation score of 0.46 (95\% CI, -0.47 to 1.38$)$.

Overall, participants responded favourably towards perceived access $(M=6.15, S D=1.14)$ and perceived 
quality $(\mathrm{M}=5.41, \mathrm{SD}=1.23)$ of LGS; $90.1 \%$ of participants reported at least 'somewhat easy' access to LGS and $76.1 \%$ of participants reported perceived quality of LGS as at least 'good'. Participants reported a mean NR score of $3.29(\mathrm{SD}=0.73)$. This is comparable to $\mathrm{NR}$ scores reported in previous literature [44, 45].

In this study, engagement with the natural environment is indicated by visit frequency to LGS. In total, $67.7 \%$ of participants reported visiting LGS at least a 'few times a month'. Active travel to LGS was reported by $85.6 \%$ of participants and the vast majority reported travel duration to LGS of less than $20 \min (86.5 \%)$. Additionally, $18.4 \%$ of participants reported actively commuting to their place of work.

In total, $75.5 \%$ of participants $(77.7 \%$ of men and $73.2 \%$ of women) reported meeting the current UK PA guidelines of at least $600 \mathrm{MET}$.min per week [41]. This is higher than national averages (66\% of men and $56 \%$ of women; [4]). Subsequently, $24.5 \%$ of participants did not complete enough MET.min per week to sustain a healthy lifestyle. Participants obtained the most amount of MET.min through walking $(\mathrm{M}=54.7 \%)$.

\section{What predicts visit frequency to LGS?}

An ordinal regression was run to predict visit frequency to LGS based on perceptions and objective measures of LGS (see Table 1). A Nagelkerke $\mathrm{R}^{2}$ of 0.226 indicates that the model explained $22.6 \%$ of the variation in visit frequency. After controlling for covariates, NR was the strongest predictor of visit frequency to LGS. An increase in NR was associated with an increase in the odds of visiting LGS more frequently (OR $=2.234,95 \% \mathrm{CI}, 1.937$ to 2.581). Perceived quality of LGS also significantly predicted visit frequency $(\mathrm{OR}=1.537,95 \% \mathrm{CI}, 1.388$ to 1.704), but perceived access did not.

Table 1 Odds ratios of visit frequency to LGS

\begin{tabular}{llccc}
\hline & & ${ }^{\mathrm{a}} \mathrm{OR}$ & \multicolumn{2}{c}{$\mathrm{b} 95 \% \mathrm{Cl}$} \\
\cline { 4 - 5 } & & & Lower & Upper \\
\hline Covariates & Age & 0.994 & 0.985 & 1.002 \\
& Health & 1.071 & 0.985 & 1.165 \\
& Gender & 1.003 & 0.832 & 1.210 \\
& \% of Road Coverage & 1.011 & 0.976 & 1.047 \\
& Environmental Deprivation & 1.082 & 0.970 & 1.206 \\
Objective & Active Travel to Work & 1.125 & 0.915 & 1.384 \\
Subjective & Perceived Local Green Space & 1.006 & 0.998 & 1.015 \\
& Perceived Quality & 1.106 & 0.994 & 1.230 \\
& Nature Relatedness & $1.537^{*}$ & 1.388 & 1.704 \\
& $2.234^{*}$ & 1.937 & 2.581 \\
\hline
\end{tabular}

Note. $R^{2}=.226$ (Cox and Snell), .226 (Nagelkerke). Model $X^{2}(10)=348.022$, $p<0.01$. ${ }^{a}$ Odds Ratios, ${ }^{b} 95 \%$ Confidence Intervals, ${ }^{*}$ indicates significance at $p<0.01$
What predicts whether participants meet PA guidelines? A binary logistic regression was run to determine which variables predicted the likelihood of meeting PA guidelines (see Table 2); the model explained $13.1 \%$ of the variance (Nagelkerke $R^{2}=0.131$ ). After controlling for covariates, NR was the only significant predictor of meeting PA guidelines ( $\mathrm{OR}=1.268,95 \% \mathrm{CI}, 1.128$ to 1.424$)$. Neither pLGS (Access and Quality) nor objectively measured green space were significant predictors. Of the covariates, subjective health and active travel to both work and LGS were significant.

\section{Does visit frequency to LGS predict whether participants meet PA guidelines?}

A binary logistic regression was run to predict the likelihood of meeting PA guidelines based upon visit frequency to LGS. The model explained $16.8 \%$ of the variation in whether participants met PA guidelines (Nagelkerke $R^{2}=$ 0.168). As illustrated by Fig. 1, as visit frequency to LGS increased so did the likelihood of achieving PA guidelines (compared to never going).

\section{Discussion}

This study found that as the number of visits to LGS increased so did the odds ratio of meeting PA guidelines. The findings also highlight the importance of NR, which was the strongest predictor of both visit frequency to LGS and meeting PA guidelines. In contrast, objectively measured quantity of LGS was not a significant predictor of visit frequency to LGS or meeting PA guidelines. Furthermore, perceived quality and perceived access did not significantly predict the likelihood of meeting PA guidelines, but perceived quality of LGS did significantly predict visit frequency.

Table 2 Odds ratios of meeting PA guidelines

\begin{tabular}{lllll}
\hline & & ${ }^{\mathrm{a}} \mathrm{OR}$ & \multicolumn{2}{l}{${ }^{\mathrm{b}} 95 \% \mathrm{Cl}$} \\
\cline { 3 - 5 } & & & Lower & Upper \\
\hline Covariates & Age & 0.994 & 0.982 & 1.006 \\
& Health & $1.268^{*}$ & 1.128 & 1.424 \\
& Gender & 0.779 & 0.601 & 1.010 \\
& \% of Road Coverage & 1.017 & 0.969 & 1.067 \\
& Environmental Deprivation & 0.997 & 0.859 & 1.158 \\
& Active Travel to Work & $1.971^{*}$ & 1.441 & 2.695 \\
& Active Travel to Local Green Space & $1.600^{*}$ & 1.076 & 2.378 \\
Objective & \% of Local Green Space & 0.994 & 0.982 & 1.006 \\
Subjective & Perceived Access & 0.993 & 0.856 & 1.151 \\
& Perceived Quality & 1.042 & 0.908 & 1.197 \\
& Nature Relatedness & $1.268^{*}$ & 1.128 & 1.424 \\
\hline
\end{tabular}

Note. $R^{2}=.089$ (Cox and Snell), .131 (Nagelkerke). Model $X^{2}(11)=125.680$, $p<0.01$. ${ }^{a}$ Odds Ratios, ${ }^{b} 95 \%$ Confidence Intervals, * indicates significance at $p<0.01$ 


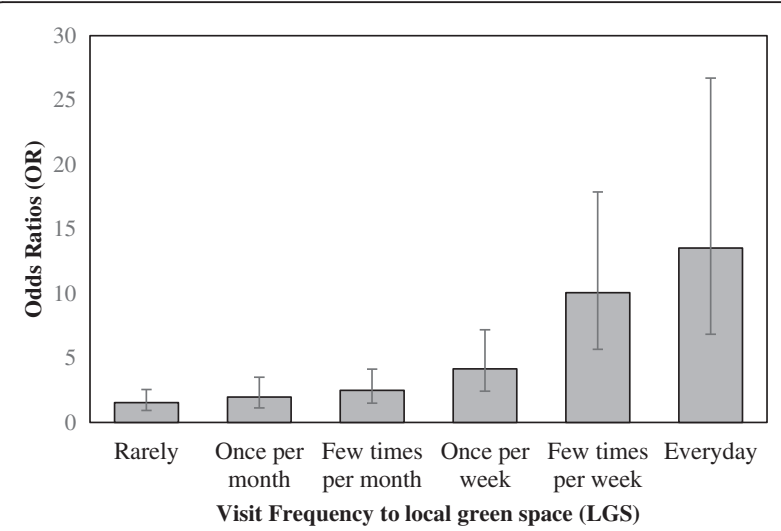

Fig. 1 Odds ratios of achieving physical activity guidelines by visit frequency to local green space (compared to never going). The odds ratios (OR) and $95 \%$ Confidence Intervals of meeting physical activity guidelines (600 MET.min per week), compared with never going to LGS

The current research was the first nationwide study to examine the relationship between LGS, visits to LGS, and PA in the UK. Previous studies (see [10] for a review) have often investigated visits to all natural or green spaces regardless of proximity to home. This study specifically asked respondents about LGS closest to home. LGS are places that are close to homes and therefore should be accessible for the majority irrespective of whether the household owns a car.

Furthermore, the current study was the first study to assess NR, pLGS, and objectively measured LGS as predictors of visit frequency to LGS and PA levels. Not only does this expand upon the existing literature regarding objectively measured LGS and PA, the findings highlight the importance of subjective variables relating to LGS.

Within this study we examined the influence of subjective measures associated with LGS on green space usage. Consistent with previous research [30] we found that NR was positively associated with visit frequency to LGS. In fact, over and above a variety of independent variables and covariates, NR was the strongest predictor of visit frequency to LGS. In doing so, our evidence supports the schematic model proposed by Calogiuri et al. [10], in which feelings about nature are related to intentions to visit LGS. It also supports the notion that visit frequency to LGS moderates the relationship between NR and psychological well-being (as highlighted by [31]). Green exercise research suggests that PA in LGS can have a positive effect on many indices associated with psychological well-being [46]. Therefore, visiting LGS more often is likely to increase psychological well-being and further investigation is warranted to assess what role NR has on this relationship.

The current findings suggest that pLGS may impact upon behaviour more than quantity of LGS. In addition to the influence of NR, perceived quality also significantly predicted visit frequency to LGS but objectively measured quantity of LGS did not. With regards to perceived quality of LGS, previous research may give an indication of how this could be enhanced, with perceived attractiveness, perceived availability of features [47] and park characteristics [48] all suggested to play an important role in the relationship between LGS and PA.

Neither perceived access nor perceived quality of LGS significantly predicted whether participants met PA guidelines. One possible explanation for this is the high percentage $(75.5 \%)$ of participants who met PA guidelines (14.5\% higher than the national average in England). In fact, males were $11.7 \%$ and females were $17.2 \%$ higher than the national average. This is most likely due to the sample being exclusively employed people. Current evidence suggests that those in formal employment were more likely to know the current recommendations for PA in the UK, and be physically active [49].

One of the main strengths of this study is the inclusion of both subjective and objective measures of LGS. Most previous studies in this area compared quantity of LGS (described as objectively measured quantity or perceived access) with PA. Our study added more robustness to this relationship with additional subjective measures. We expanded the limited research on NR and have shown its importance in the relationship between LGS and PA.

Against these contributions, some limitations should be noted. First, due to the correlational nature of the study, causality cannot be inferred in the observed relationships. Second, the study used a self-reported measure of PA. Although the IPAQ is well used in the literature, people often over-estimate PA levels [50]. Furthermore, this study did not explore PA in detail. Had we also explored 'green exercise', as opposed to just overall PA levels, we may have been able to provide stronger explanations for the results. Further investigation of green exercise, distinct from $\mathrm{PA}$, is warranted to provide better understanding of the mechanisms between LGS, PA and health.

One further limitation is the double inclusion of objectively measured LGS: the environmental deprivation score - that was used as a covariate - was calculated in part using objectively measured LGS. This was deemed necessary as it included a variety of additional factors such as climate, and pollution etc.. ${ }^{2}$ Although efforts were made to account for environmental factors, the level of detail required to accurately portray the favourableness of home location for green exercise was beyond the reach of this study. For example, street lighting and pedestrian pathways that link housing areas to LGS may influence visit frequency.

As mentioned previously, the inclusion of only employed individuals does limit the ability to generalise the findings to other populations e.g. unemployed, retired. Likewise, whilst active commuters were controlled for in statistical 
analysis, this analysis did not explore visiting green space during work hours, and the subsequent impact this may have had on PA levels. Additionally, we did not account for variations in employment type. Further work is needed to explore how the complexities of working life (location, activity levels, environment etc.) influence the relationships we found.

Results from this study show that on average participants had less LGS than the national average at ward level. Even though the percentage of employed people is about the same for rural and urban areas in England, the vast majority of people in England live in urban areas (81.5\% of people in 2011). We suggest that the inclusion of only employed participants skewed the results towards more urbanised wards. It is therefore likely that the majority of participants reported visits to urban green space rather than natural environments, although we do not have the data to confirm this.

\section{Conclusion}

This is the first nationwide study to explore the relationship between LGS and PA. We found that visit frequency to LGS is associated with the likelihood of meeting PA guidelines. Furthermore, subjective measures of LGS, and particularly NR, appear to be more important than objectively measured quantity of LGS for predicting both visit frequency to LGS and PA. As PA is known to have many positive health benefits, visits to LGS, especially if active transport is used, potentially could have a significant impact on Public Health.

\section{Availability of data and materials}

The dataset supporting the conclusions of this article is available in the UK Data Service repository http://reshar e.ukdataservice.ac.uk/852253/.

\section{Endnotes}

${ }^{1}$ Road coverage for Scotland, Wales, and Northern Ireland was recorded as missing data and treated as such in the analyses. The analyses were re-run including only participants from England and the same pattern of results was observed. As such, results from the full sample are reported in the manuscript.

${ }^{2}$ Statistical analysis revealed that when environmental deprivation was removed as a covariate, objectively measure quantity of LGS was not a significant predictor of visit frequency to LGS or PA. Therefore, environmental deprivation - as a covariate - was included in the main analysis.

\section{Abbreviations}

LGS: local green space; MET.min: MET minutes; METs: multiples of the resting metabolic rate; NR: nature relatedness; PA: physical activity;

pLGS: perceptions of local green space.

\section{Competing interests}

The authors declare that they have no competing interests.

\section{Authors' contributions}

EF participated in the design of the study, carried out the statistical analyses, interpreted the data and drafted the manuscript. VG was involved with design of the study, data collection, statistical analyses, and critically revised the manuscript. PF was involved with statistical analyses, interpretation of data and critically revised the manuscript. All authors read and approved the manuscript. Also, all authors agree to be accountable for all aspects of the work in ensuring that questions related to the accuracy or integrity of any part of the work are appropriately investigated and resolved.

\section{Acknowledgements}

The authors would like to thank Dr Jo Barton from the University of Essex, Green Exercise Research Group and Professor Peter Lynn from the Institute of Social and Economic Research, University of Essex. This research was funded by the Economic and Social Research Council (Project Number RES-064-27-0019)

Received: 3 September 2015 Accepted: 29 April 2016

Published online: 20 May 2016

\section{References}

1. Bouchard C, Blair SN, Haskell W. Physical Activity and Health-2nd Edition: Human Kinetics $10 \% .2006$.

2. NHS. Physical activity guidelines for adults 2015 [updated 11/07/2015; cited 2015 1st June]. Available from: http://www.nhs.uk/Livewell/fitness/Pages/ physical-activity-guidelines-for-adults.aspx.

3. US Department of Health and Human Services. 2008 Physical Activity Guidelines for Americans - Summary 2015 [cited 2015 1st June]. Available from: http://health.gov/paguidelines/guidelines/summary.aspx.

4. HSE. Health Survey for England 2012. Chapter 2, Physical activity in adults: Health \& Social Care Information Centre; 2013 [cited 2015 1st June]. Available from: http://www.hscic.gov.uk/catalogue/PUB13218.

5. WHO. Prevalence of insufficient physical activity 2015 [cited 2015 1st June]. Available from: http://www.who.int/gho/ncd/risk_factors/physical_activity_ text/en/.

6. WHO. New physical activity recommendations for reducing disease and prevent deaths 2015 [cited 2015 1st June]. Available from: http://www.who. int/chp/media/news/releases/2011_2_physicalactivity/en/.

7. Kaczynski AT, Henderson KA. Environmental Correlates of Physical Activity: a review of evidence about parks and recreation. Leis Sci. 2007:29(4):315-54.

8. Lee AC, Maheswaran R. The health benefits of urban green spaces: a review of the evidence. J Public Health (Oxf). 2011;33(2):212-22.

9. Moran M, Van Cauwenberg J, Hercky-Linnewiel R, Cerin E, Deforche B, Plaut $P$. Understanding the relationships between the physical environment and physical activity. Int J Behav Nutr Phys Act. 2014;11:79.

10. Calogiuri G, Chroni S. The impact of the natural environment on the promotion of active living: an integrative systematic review. BMC Public Health. 2014;14:873.

11. England N. 'Nature Nearby' Accessible Natural Greenspace Guidance. 2010.

12. D'Haese S, De Meester F, Cardon G, De Bourdeaudhuij I, Deforche B, Van Dyck $D$. Changes in the perceived neighborhood environment in relation to changes in physical activity: a longitudinal study from childhood into adolescence. Health Place. 2015;33:132-41.

13. Edwards P, Tsouros AD. Promoting Physical Activity and Active Living in Urban Environments: The Role of Local Governments: WHO Regional Office for Europe. 2006.

14. Giles-Corti B, Broomhall MH, Knuiman M, Collins C, Douglas K, Ng K, et al. Increasing walking: how important is distance to, attractiveness, and size of public open space? Am J Prev Med. 2005;28(2):169-76.

15. Coutts C, Chapin T, Horner M, Taylor C. County-level eftects of green space access on physical activity. J Phys Act Health. 2013;10:232-40.

16. Khalil R. Quantitative evaluation of distribution and accessibility of urban green spaces (Case study: City of Jeddah). Int J of Geom \& Geos. 2014;4(3): $526-35$

17. Shanahan DF, Lin BB, Gaston KJ, Bush R, Fuller RA. Socio-economic inequalities in access to nature on public and private lands: a case study from Brisbane, Australia. Landsc Urban Plan. 2014;130:14-23. 
18. Bai H, Wilhelm Stanis SA, Kaczynski AT, Besenyi GM. Perceptions of neighborhood park quality: associations with physical activity and body mass index. Ann Behav Med. 2013;45 Suppl 1:S39-48.

19. Hillsdon M, Jones A, Coombes E. Green space access, green space use, physical activity and overweight, Natural England Commissioned Reports. 2011.

20. Lackey KJ, Kaczynski AT. Correspondence of perceived vs. objective proximity to parks and their relationship to park-based physical activity. Int J Behav Nutr Phys Act. 2009;6:53.

21. Leslie E, Sugiyama T, lerodiaconou D, Kremer P. Perceived and objectively measured greenness of neighbourhoods: Are they measuring the same thing? Landsc Urban Plan. 2010;95(1-2):28-33.

22. Maas J, Verheij RA, de Vries S, Spreeuwenberg P, Schellevis FG, Groenewegen PP. Morbidity is related to a green living environment. J Epidemiol Community Health. 2009;63(12):967-73.

23. Mytton OT, Townsend N, Rutter H, Foster C. Green space and physical activity: an observational study using Health Survey for England data. Health Place. 2012;18(5):1034-41.

24. Witten K, Hiscock R, Pearce J, Blakely T. Neighbourhood access to open spaces and the physical activity of residents: a national study. Prev Med. 2008;47(3):299-303.

25. Ord K, Mitchell R, Pearce J. Is level of neighbourhood green space associated with physical activity in green space. Int J Behav Nutr Phys Act. 2013;10:127.

26. McGinn AP, Evenson KR, Herring AH, Huston SL. The relationship between leisure, walking, and transportation activity with the natural environment. Health Place. 2007;13(3):588-602.

27. Veitch J, Carver A, Abbott G, Giles-Corti B, Timperio A, Salmon J. How active are people in metropolitan parks? An observational study of park visitation in Australia. BMC Public Health. 2015;15:610.

28. Kirri A, Gardiner J, Huang C. Quality of Life Survey 2014: Report on Oxford's 7 priority neighbourhoods. 2014.

29. Van Cauwenberg J, Cerin E, Timperio A, Salmon J, Deforche B, Veitch J. Park proximity, quality and recreational physical activity among mid-older aged adults: moderating effects of individual factors and area of residence. Int J Behav Nutr Phys Act. 2015;12(1):46.

30. Lin BB, Fuller RA, Bush R, Gaston KJ, Shanahan DF. Opportunity or orientation? Who uses urban parks and why. PLoS One. 2014;9(1):e87422.

31. Zhang JW, Howell RT, lyer R. Engagement with natural beauty moderates the positive relation between connectedness with nature and psychological well-being. J Environ Psychol. 2014;38:55-63.

32. Zelenski JM, Nisbet EK. Happiness and feeling connected: the distinct role of nature relatedness. Environ Behav. 2012;46(1):3-23.

33. Office of National Statistics. 2011 Census Analysis - Comparing Rural and Urban Areas of England and Wales. 2011.

34. Met Office. 2011 weather summaries 2014 [cited 2016 1st February] Available from: http://www.metoffice.gov.uk/climate/uk/summaries/2011.

35. Office of National Statistics. All people (2001 census). 2001.

36. Richardson EA, Mitchell R. Gender differences in relationships between urban green space and health in the United Kingdom. Soc Sci Med. 2010;71(3):568-75.

37. CORINE land cover [Internet]. 2000. Available from: http://www.eea.europa. eu/publications/CORO-landcover.

38. Nisbet EK, Zelenski JM, Murphy SA. The Nature Relatedness Scale: linking individuals' connection with nature to environmental concern and behavior. Environ Behav. 2008;41(5):715-40.

39. Booth ML, Ainsworth BE, Pratt M, Ekelund U, Yngve A, Sallis JF, et al. International physical activity questionnaire: 12-country reliability and validity. Med Sci Sports Exerc. 2003;195(9131/03):3508-1381.

40. The IPAQ Group. Guidelines for the data processing and analysis of the "International Physical Activity Questionnaire". 2005.

41. Department of Health. Start Active, Stay Active. 2011.

42. Richardson EA, Mitchell R, Shortt NK, Pearce J, Dawson TP. Developing summary measures of health-related multiple physical environmental deprivation for epidemiological research. Environ Plan. 2010;42:1650-68.

43. Office of the Deputy Prime Minister. Generalised land use database statistics for England. London: ODPM Publications; 2001

44. Martyn P, Brymer E. The relationship between nature relatedness and anxiety. J Health Psychol. 2014. Epub ahead of print.

45. Nisbet EK, Zelenski JM. The NR-6: a new brief measure of nature relatedness. Front Psychol. 2013:4:813.
46. Barton J, Pretty J. What is the best dose of nature and green exercise for improving mental health? A multi-study analysis. Environ Sci Technol. 2010:44(10):3947-55.

47. Kaczynski AT, Besenyi GM, Stanis SA, Koohsari MJ, Oestman KB, Bergstrom R, et al. Are park proximity and park features related to park use and parkbased physical activity among adults? Variations by multiple sociodemographic characteristics. Int J Behav Nutr Phys Act. 2014;11:146.

48. Ries AV, Voorhees CC, Roche KM, Gittelsohn J, Yan AF, Astone NM. A quantitative examination of park characteristics related to park use and physical activity among urban youth. J Adolesc Health. 2009:45(3 Suppl):S64-70

49. Farrell L, Hollingsworth B, Propper C, Shields MA. The socioeconomic gradient in physical inactivity: evidence from one million adults in England. Soc Sci Med. 2014;123:55-63.

50. Rzewnicki R. Health-enhancing physical activity measurement and determinants of daily activity at home, work, travel, and leisure. Leuven: KU Leuven; 2003.

\section{Submit your next manuscript to BioMed Central and we will help you at every step:}

- We accept pre-submission inquiries

- Our selector tool helps you to find the most relevant journal

- We provide round the clock customer support

- Convenient online submission

- Thorough peer review

- Inclusion in PubMed and all major indexing services

- Maximum visibility for your research

Submit your manuscript at www.biomedcentral.com/submit

) Biomed Central 